\title{
Icterícia neonatal: atuação do enfermeiro frente à identificação precoce e tratamento
}

\author{
Neonatal jaundice: nurses' action against early identification \\ Ictericia neonatal: acción de las enfermeiras contra la identificación y el tratamento temprano
}

\author{
Camila Domingues Godoy \\ ORCID: https://orcid.org/0000-0002-1522-7828 \\ Faculdade Pitágoras de Londrina, Brasil \\ E-mail: camila.godoy@outlook.com \\ Mykhaela Misael de Aguiar e Silva \\ ORCID: https://orcid.org/0000-0002-1469-9145 \\ Faculdade Pitágoras de Londrina, Brasil \\ E-mail: mykhaelamisael@gmail.com \\ Thais Cristina dos Santos \\ ORCID: https://orcid.org/0000-0002-6785-7485 \\ Faculdade Pitágoras de Londrina, Brasil \\ E-mail: thais.c.santos_enf@outlook.com \\ Cleiton José Santana \\ ORCID: https://orcid.org/0000-0002-8150-2357 \\ Faculdade Pitágoras de Londrina, Brasil \\ E-mail:cleitonjsantana@hotmail.com \\ Ludmilla Laura Miranda \\ ORCID: https://orcid.org/0000-0002-8056-5551 \\ Universidade Estadual de Londrina, Brasil \\ E-mail: m.ludmilla@hotmail.com
}

\begin{abstract}
Resumo
Icterícia neonatal é a presença anormal de pigmentos biliares, podendo apresentar etiologia fisiológica ou patológica. A terapêutica para a icterícia é variada, a intervenção mais utilizada é a fototerapia, sendo a equipe de enfermagem responsável pelo preparo e acolhimento do bebê, orientar os pais e prestar os cuidados necessários para prevenir-se das possíveis consequências desse tratamento. O objetivo foi descrever a atuação do enfermeiro na identificação precoce da icterícia neonatal e a assistência de enfermagem durante o tratamento com uso da fototerapia. Tratou-se de uma revisão bibliográfica, com caráter descritivo e qualitativo, utilizando as bases de dados Scielo, Lilacs, BVS e BDENF. Destaca-se a existência de dois tipos de icterícia, sendo a fisiológica que ocorre devido à imaturidade do fígado para a excreção da bilirrubina quando níveis séricos excede $25 \mathrm{mg} / \mathrm{dl}$ e a patológica que não é caracterizada somente pelos níveis séricos da bilirrubina. A icterícia neonatal é um diagnóstico de enfermagem comum na primeira semana de vida dos recém-nascidos, podendo ser responsável por várias reinternações necessitando de intervenções precoces e eficazes. O diagnóstico e tratamento precoce da hiperbilirrubinemia neonatal é de suma importância para a prevenção de sua principal complicação o kernicterus. Para do tratamento adequado deve-se observar o histórico materno e neonatal, o aparecimento e a evolução da icterícia através do exame físico minucioso no bebê e a coleta de sangue para análise, garantindo assim uma terapêutica mais segura. Por fim, determinar em qual tipo hiperbilirrubinemia enquadra-se e utilizar a intervenção mais eficiente no tratamento e prevenção da icterícia.
\end{abstract}

Palavras-chave: Icterícia neonatal; Cuidados de enfermagem; Fototerapia; Hiperbilirrubinemia.

\begin{abstract}
Neonatal jaundice is the abnormal presence of biliary pigments, which may have a physiological or pathological etiology. The treatment for jaundice is varied, the most used intervention is phototherapy, with the nursing team being responsible for preparing and welcoming the baby, guiding parents and providing the necessary care to prevent the possible consequences of this treatment. The objective was to describe the role of nurses in the early identification of neonatal jaundice and nursing care during treatment using phototherapy. It was a bibliographic review, with a descriptive and qualitative character, using the Scielo, Lilacs, BVS and BDENF databases. The existence of two types of jaundice is noteworthy, the physiological one that occurs due to the immaturity of the liver for the excretion of bilirubin when serum levels exceed $25 \mathrm{mg} / \mathrm{dl}$ and the pathological one, which is not characterized only by the serum levels of bilirubin. Neonatal jaundice is a common nursing diagnosis in the first week of life of newborns and may be responsible for several readmissions requiring early and effective interventions. Early diagnosis and treatment of neonatal hyperbilirubinemia is of paramount importance for the prevention of its main complication, kernicterus. For proper treatment, maternal and neonatal history, the appearance and evolution of jaundice must be observed through a thorough physical examination of the baby and blood collection for analysis, thus ensuring a safer therapy. Finally,
\end{abstract}


determine which type of hyperbilirubinemia fits and use the most efficient intervention in the treatment and prevention of jaundice.

Keywords: Neonatal jaundice; Nursing care; Phototherapy; Hyperbilirrubinemia.

\begin{abstract}
Resumen
La ictericia neonatal es la presencia anormal de pigmentos biliares, que pueden tener una etiología fisiológica o patológica. El tratamiento de la ictericia es variado, la intervención más utilizada es la fototerapia, siendo el equipo de enfermería el encargado de preparar y acoger al bebé, orientar a los padres y brindar los cuidados necesarios para prevenir las posibles consecuencias de este tratamiento. El objetivo fue describir el papel de las enfermeras en la identificación precoz de la ictericia neonatal y los cuidados de enfermería durante el tratamiento mediante fototerapia. Se trató de una revisión bibliográfica, de carácter descriptivo y cualitativo, utilizando las bases de datos Scielo, Lilacs, BVS y BDENF. Destaca la existencia de dos tipos de ictericia, la fisiológica que se produce por la inmadurez del hígado para la excreción de bilirrubina cuando los niveles séricos superan los $25 \mathrm{mg} / \mathrm{dl}$ y la patológica, que no se caracteriza solo por los niveles séricos de bilirrubina. La ictericia neonatal es un diagnóstico de enfermería común en la primera semana de vida de los recién nacidos y puede ser responsable de varios reingresos que requieren intervenciones tempranas y efectivas. El diagnóstico y tratamiento precoces de la hiperbilirrubinemia neonatal es de suma importancia para la prevención de su principal complicación, el kernicterus. Para un tratamiento adecuado, se deben observar los antecedentes maternos y neonatales, la aparición y evolución de la ictericia mediante un examen físico minucioso del bebé y una extracción de sangre para su análisis, garantizando así una terapia más segura. Por último, determine qué tipo de hiperbilirrubinemia se ajusta y utilice la intervención más eficaz en el tratamiento y la prevención de la ictericia.
\end{abstract}

Palabras clave: Ictericia neonatal; Cuidado de enfermera; Fototerapia; Hiperbilirrubinemia.

\title{
1. Introdução
}

Entende-se como icterícia neonatal, a presença anormal de pigmentos biliares, a qual pode apresentar etiologia fisiológica ou patológica sendo que o seu tratamento dependerá da causa e da intensidade. Sendo caracterizado como uma das modificações mais presentes em recém-nascidos (Sacramento et. al., 2017; Guedes Junior, 2019).

Segundo Sacramento e colaboradores (2017) a bilirrubina é conceituada como um composto proveniente da degradação da hemoglobina resultante da destruição das hemácias, sendo que, durante a gestação, este composto é filtrado pela placenta e excretado pelo fígado da mãe, consequentemente após o nascimento, demanda-se que o fígado do bebê realize esse processo sozinho. Sabe-se que os recém-nascidos em sua maioria, apresentam icterícia, entretanto, se expressa cautela quanto ao evento, uma vez que, a bilirrubina apresenta toxicidade ao sistema nervoso. A presença deste composto em excesso acarreta na coloração amarela da pele, mucosas e secreções e isso, define-se por icterícia (Santos, 2016; Guedes Junior, 2019).

A terapêutica para a icterícia é amplamente variada, contudo, a intervenção mais utilizada é a fototerapia, sendo a equipe de enfermagem responsável pelo preparo e acolhimento do bebê para o tratamento, sendo atribuição dos profissionais orientarem os pais quanto ao quadro clínico, instalarem o equipamento, bem como, prestar os cuidados necessários, como a proteção ocular, a distância e o posicionamento da fonte luminosa, para prevenir-se das possíveis consequências desse tratamento (Machado, Samico, Brega, 2012; Sena, Reis, Cavalcante, 2015).

Assim, a presente pesquisa se justifica com base na necessidade de conhecer a importância da atuação do profissional de saúde, enfermeiro, em frente ao diagnóstico precoce, bem como o esclarecimento dos tratamentos desta patologia. A motivação para sustentar este estudo reside na importância do tema para a sociedade, e para a formação de enfermeiros, contribuindo com o tema apontando a necessidade dessa forma de atuação, para esses profissionais de saúde. Dessa forma, o objetivo da pesquisa consiste em descrever a atuação do enfermeiro na identificação precoce da icterícia neonatal e a assistência de enfermagem durante o tratamento com uso da fototerapia. 


\section{Metodologia}

Este estudo, trata-se de uma revisão bibliográfica, com caráter descritivo e qualitativo, a qual apresenta ampla abordagem metodológica, proporcionando a síntese das evidências disponíveis acerca do tema pesquisado. Buscou-se responder a seguinte questão: o enfermeiro está qualificado para realizar o diagnóstico precoce de icterícia neonatal durante o exame físico do recém-nascido bem como oferecer uma assistência de enfermagem durante o tratamento?

As buscas foram desenvolvidas a partir de estudos científicos indexados nas bases de dados eletrônico Scientific Electronic Library Online (SCIELO), Centro Latino-Americano e do Caribe de Informação em Ciências da Saúde (LILACS), Biblioteca Virtual de Saúde (BVS) e Base de Dados em enfermagem (BDENF).

Para a amostra da revisão bibliográfica, utilizou-se estudos científicos disponibilizados na íntegra e na forma online, estabeleceu como critérios de inclusão, artigos científicos na íntegra e na forma online, publicados no idioma português entre o período de 2010 a 2020 e que incluam os descritores selecionados. Como critério de exclusão, determina-se os relatos de casos informais, reportagens, notícias, editoriais, textos não científicos e estudos científicos sem disponibilidade do texto na íntegra online e que não contemplaram os critérios de inclusão.

Sendo que, utilizou-se as palavras registradas nos Descritores em Ciências da Saúde (DeCS) para realizar as buscas dos estudos nas bases de dados, sendo elas "icterícia neonatal”; "cuidados de enfermagem"; "hiperbilirrubinemia neonatal", posteriormente realizou-se uma leitura analítica para ordenar as informações.

Foram encontrados 38 artigos no total e a extração dos dados ocorreu a partir dos critérios de inclusão: idiomas selecionados, artigo da temática e que respondesse à pergunta de pesquisa. Após a leitura na íntegra, foram utilizados 23 no estudo.

\section{Resultados e Discussão}

\section{Icterícia Neonatal}

A hiperbilirrubinemia conhecida como icterícia, constitui-se como uma síndrome frequente durante o período neonatal, tendo como característica processos fisiológicos e/ou patológicos, com apresentação da elevação de bilirrubina no sangue e consequentemente manifesta-se a coloração amarelada da pele, mucosa e líquidos orgânicos (Sousa, Sena, 2012; Nóbrega Junior, Vieira, Guedes Junior, 2019).

Entende-se que a etiologia desta patologia é multifatorial, entre eles, fatores maternos e perinatais, dos quais, destacam-se a idade gestacional (IG), sexo do recém-nascido (RN), diabetes mellitus gestacional (DMG), pré-eclâmpsia, tempo de clampeamento do cordão umbilical, eliminação tardia de mecônio, nutrição enteral tardia e perda de peso importante na primeira semana de vida. Sendo que, situações específicas ocasionam o aumento da circulação êntero-hepática, como por exemplo o jejum prolongado e anomalias gastrointestinais (processos obstrutivos, estenose hipertrófica do piloro) e favorecem a hiperbilirrubinemia. Destaca-se também como fatores importantes, doenças hemolíticas (incompatibilidades ABO e Rh) e a presença de coleções sanguíneas extravasculares (cefalohematoma, bossa serossanguínea e equimoses) (Carvalho e Lavor, 2020).

Segundo Pinto (2014) a icterícia se expressa quase sempre benigna, entretanto, quando se encontra elevada, pode acarretar danos ao sistema nervoso dos recém-nascidos (RN), desta forma ocorre um acompanhamento ambulatorial inadequado devido à precocidade das altas. Sendo assim, aumenta-se a probabilidade de hiperbilirrubinemia patológica, uma vez que, os sinais são despercebidos pelos familiares por não possuírem habilidades para avaliar tal evento e consequentemente, evolui-se para sequelas graves. 
Destaca-se a existência de dois tipos de icterícia, sendo a fisiológica que ocorre devido à imaturidade do fígado para a excreção da bilirrubina, definida pelos níveis séricos quando a mesma excede $25 \mathrm{mg} / \mathrm{dl}$ e a patológica não é caracterizada somente pelos níveis séricos da bilirrubina (Sena, Reis, Cavalcante, 2015).

De acordo com o Romano (2017) a hiperbilirrubinemia deve-se à elevação do turnover eritrocitário associado à imaturidade hepática do recém-nascido, ocasionando um desnivelamento entre a produção e a conjugação de bilirrubina. Sendo caracterizada como uma situação patológica quando surge nas primeiras 24 horas ou se mantém por mais de 2 semanas de vida e quando os níveis de bilirrubina metabolizada ultrapassam os $20 \%$ da bilirrubina total.

A icterícia neonatal ocorre devido ao acúmulo do pigmento bilirrubínico não conjugado, sendo que em torno de 80 a 90\% da bilirrubina decorre da síntese da hemoglobina ou de eritropoiese ineficaz. Sendo assim, a bilirrubina é produto do catabolismo do heme. A enzima heme oxigenase, realiza a catalisação da quebra do heme decorrente da biliverdina que rapidamente se transforma em bilirrubina indireta por ação da biliverdina redutase, esta qual, liga-se a albumina para ser transportada para o fígado e ao chegarem ao hepatócito ocorre uma desassociação para que a bilirrubina indireta seja absorvida e processada. Posteriormente, a enzima uridina difosfogluconurato glucoronosiltransferase (UFT1A1) promove a conjugação da bilirrubina com o ácido glucorônico produzindo a bilirrubina direta, a qual possui a característica de ser hidrossolúvel, sendo assim, mais facilmente excretada pelo sistema biliar e trato gastrointestinal (Carvalho \& Almeida, 2020).

Estudos apontam que no organismo do RN o metabolismo da bilirrubina encontra-se em transição, entre a fase fetal e a fase adulta, sendo necessária a atuação de uma enzima hepática para sintetizar a bilirrubina indireta (BI), que é lipossolúvel, em bilirrubina direta (BD), substância hidrossolúvel, entretanto, esta enzima possui baixa atividade neste período e encontra-se também uma sobrecarga hepática, uma vez que, há uma maior degradação da hemoglobina fetal (Di Sarli, 2018).

A bilirrubina atua como antioxidante nesta fase da vida, beneficiando o organismo do RN, entretanto, em situações de icterícia patológica ocasiona-se riscos de ocorrer impregnação cerebral, ressaltando a necessidade da identificação precoce e tratamento imediato. Sendo o diagnóstico realizado com embasamento na história clínica, no exame físico e análise dos níveis séricos (Sacramento et. al., 2017).

\section{Metabolismo da bilirrubina}

A bilirrubina é o produto final da degradação do grupo heme. Sendo que, em torno de 70-80\% da bilirrubina produzida diariamente deriva-se da degradação da hemoglobina dos glóbulos vermelhos maturados. A sua formação acontece no baço e no fígado, diante exposto, na primeira reação, catalisada pela enzima heme oxigenase quebra a ponte alfa do grupo da porfirina e abre o anel do grupo heme, em seguida, os produtos finais dessa reação são a biliverdina, o monóxido de carbono e o ferro; na segunda reação catalisada pela enzima biliverdina redutase, ocorre a síntese da ponte central de metileno de biliverdina, convertendo-a em bilirrubina ( Di Sarli, 2018; Ferreira, 2018).

A bilirrubina é insolúvel em água, sendo assim, é transportada no sangue até o fígado, a bilirrubina é solubilizada através da ligação reversível e não covalente à albumina. No fígado, a bilirrubina realiza uma dissociação da albumina e assim é captada e internalizada pelos hepatócitos através de proteínas de membrana (membrane-associated organic anion transport proteins - OATPS) (Ferreira, 2018).

No hepatócito, a bilirrubina liga-se a proteínas citoplasmáticas da glutationa-S-transferase, essas proteínas atuam tanto para reduzir o fluxo de bilirrubina novamente para o plasma, bem como, também apresentam a bilirrubina para a reação de conjugação. Posteriormente, a bilirrubina é solubilizada pela ligação com o ácido glucurônico, se tornando hidrossolúvel. Essa reação de conjugação é catalisada pela uridina difosfato glucuronosiltransferase (UDP-GT) (Ferreira, 2018; Junior,Vieira, Junior, 2019; Carvalho e Lavor, 2020). 
A bilirrubina conjugada (BC) excretada para a bílis é drenada para o duodeno e se mantém inalterada ao longo do intestino delgado proximal, não sendo reabsorvida pela mucosa intestinal. Quando a BC chega ao íleo distal e ao cólon, ocorre a hidrolisação através das $\beta$-glucuronidases bacterianas em bilirrubina não conjugada (BNC), que pode voltar à circulação através da circulação entero-hepática. A BNC é degradada pelas bactérias intestinais para formar urobilinogênios. Cerca de 80-90\% destes produtos são excretados nas fezes ou sofrem oxidação em urobilinas. Os restantes 10-20\% dos urobilinogênios são reabsorvidos passivamente, entram no sangue venoso e são re-excretados pelo fígado. Uma pequena fracção (geralmente $<3 \mathrm{mg} / \mathrm{dL}$ ) escapa à captação hepática, sendo filtrada no glomérulo e excretada na urina (Romano, 2017; Ferreira, 2018).

\section{Diagnóstico precoce}

O diagnóstico de enfermagem é conceituado como um julgamento clínico sobre uma resposta humana a condições de saúde/processos da vida, ou uma suscetibilidade a essa resposta por indivíduo, família, grupo ou comunidade. Requer uma avaliação de enfermagem para o diagnóstico correto do paciente, uma vez que não é possível padronizar diagnósticos de enfermagem com segurança usando um diagnóstico médico. Diante exposto, o sistema de classificação dos diagnósticos de enfermagem mais utilizado mundialmente é a North American Nursing Diagnosis Association - NANDA - I. Baseados nessa classificação, os profissionais de saúde, enfermeiros atuam nas suas diversas áreas na busca pela assistência de excelência, com enfoque no cuidado individualizado oferecido a cada paciente (Alves, et. al., 2020).

Conforme disposto na resolução COFEN - 272/2002, A Sistematização da Assistência de Enfermagem (SAE) é uma incumbência privativa do enfermeiro passível de aplicação a partir da escolha de uma teoria do processo de enfermagem que irá direcionar as etapas da sistematização da assistência (anamnese e exame físico, diagnóstico de enfermagem, intervenções de enfermagem, resultados esperados e evolução) e fornecer estrutura para a tomada de decisões. (Teixeira, 2014).

Sendo assim, o cuidar proporciona o surgimento dos métodos e instrumentos do trabalho da enfermagem. Uma das metodologias utilizadas pelo enfermeiro para implantação e operacionalização do cuidado é a SAE o qual possibilita um cuidado organizado, sistematizado, contínuo e seguro ao RN. O cuidado de enfermagem em neonatologia é o meio pelo qual se conduz a recuperação, adaptação e o bem-estar, sendo que, este cuidado encontra-se fundamentado em conhecimentos científicos e na autonomia do profissional de enfermagem. Diante do exposto, a SAE é aplicada na prática por meio do Processo de Enfermagem (PE). Esta é a representação maior do método científico na enfermagem, por meio do qual o enfermeiro irá desenvolver e organizar o trabalho da sua equipe. O PE é um instrumento metodológico em que o enfermeiro irá organizar e favorecer o cuidado de enfermagem assegurando ao enfermeiro e sua equipe uma forma sistemática de cuidar (Moraes Filho, et. al., 2017).

A atuação do enfermeiro se tem início na detecção precoce da icterícia, através do exame físico do RN e se estende durante a terapia proposta. Assim, a equipe de enfermagem é participativa desde o recebimento e preparação do recém-nascido para a terapêutica. Sendo que deve ser levado em consideração nesse diagnóstico e terapêutica a importância da humanização durante todo o processo por meio do profissional de enfermagem que assiste (Alves, 2020).

Diante do exposto, compreende-se que a Icterícia neonatal é um diagnóstico de enfermagem comum na primeira semana de vida dos recém-nascidos, podendo ser responsável por várias reinternações e que requer intervenções de enfermagem precoces e eficazes. Assim, o diagnóstico e tratamento precoce da Hiperbilirrubinemia Neonatal é de suma importância para a prevenção de sua principal complicação o kernicterus, uma síndrome neurológica causada devido a deposição de bilirrubina nas células cerebrais que surgem quando os níveis de bilirrubina são maiores que $25 \mathrm{mg} / \mathrm{dl}$, com evidência de lesão neuronal e sequelas motoras graves (Gomes, Teixeira, Barrichello, 2010; Dantas, 2017).

Sabe-se então que o diagnóstico da icterícia neonatal através da história clínica torna-se um parâmetro de suma importância, uma vez que colhe dados sobre o histórico e fatores epidemiológicos, pois permitem detectar a possibilidade de 
desenvolvimento de hiperbilirrubinemia significante na primeira semana de vida. Dessa forma, a atuação do enfermeiro junto ao RN portador de hiperbilirrubinemia deve iniciar-se na detecção precoce da patologia que acontece durante o exame físico e estendendo-se durante a terapia proposta. Sendo assim, é necessário que os enfermeiros sejam qualificados e capazes de realizar o diagnóstico clínico de icterícia bem como proporcionar adequada assistência de enfermagem durante o tratamento (Sacramento, et. al., 2017).

Para o diagnóstico, Sarli (2018) descreve a necessidade de solicitar a dosagem de bilirrubina sérica e a aferição transcutânea (BTC), uma vez que a análise visual como parâmetro de diagnóstico pode ser subestimado, visto que depende da experiência do profissional de saúde, do tipo e da pigmentação da pele do RN e da luminosidade do ambiente.

Na realização do exame físico, a sua detecção depende principalmente da análise da pigmentação da pele do recémnascido sendo que icterícia por hiperbilirrubinemia indireta apresenta progressão céfalo-caudal. Sendo que, no que diz respeito aos exames, deve-se realizar rotineiramente a dosagem sérica da bilirrubina que pode ser feita através do hemograma, o padrão ouro para o diagnóstico, pois apresenta um resultado mais fidedigno em relação ao bilirrubinômetro transcutâneo, apesar desse equipamento fornecer as informações de forma instantânea e indolor (Sacramento, et. al., 2017).

Segundo Britto (2016) após a coleta da anamnese, os dados laboratoriais são muito importantes, em grande maioria serve como confirmação de um pré diagnóstico clínico. Sendo assim, se torna importante colher a amostra para os exames apenas $1 \mathrm{vez}$, com a finalidade de não comprometer a integridade física do RN. Contudo, a exceção existe caso haja alterações e precise de mais controle. Sendo que, os exames laboratoriais mais importantes são: Hematócrito e hemoglobina; Hemograma com reticulócitos; Coombs direto; Tipagem sanguínea e fator Rh; Dosagem de G6PD (Britto, 2016).

No que diz respeito ao diagnóstico, Gomes, Teixeira e Barrichello (2010), descrevem que:

"A detecção da hiperbilirrubinemia ocorre inicialmente, pela observação da coloração amarelada da pele do RN no exame físico de rotina, notando-se principalmente na face do RN quando os níveis séricos de bilirrubina total ultrapassam 5mg/dl. A icterícia manifesta-se progressivamente no sentido céfalo-caudal, sendo possível quantificar os níveis séricos de acordo com as regiões acometidas. Quando os níveis séricos de bilirrubina total estão até $12 \mathrm{mg} / \mathrm{dl}$ a icterícia está presente na face e tronco, já níveis maiores que $15 \mathrm{mg} / \mathrm{dl}$ há icterícia de pés e mãos. A realização de um exame físico detalhado do RN ictérico auxilia no diagnóstico correto de sua etiologia e na orientação terapêutica. Porém, a coleta de exames laboratoriais (dosagem sérica da bilirrubina total) orienta o diagnóstico ou estabelecem o tratamento a ser realizado" (Gomes, Teixeira, Barrichello, 2010).

Sabe-se que em 1969 Kramer observou através de estudos que era possível quantificar os níveis séricos de bilirrubina, e assim montou a tabela (Figura 1) para que o profissional de saúde possa observar e estabelecer os cuidados e tratamentos, esse método foi elaborado com intuito de melhorar o atendimento aos RNs com Icterícia Neonatal (Britto, 2016).

Figura 1 - Tabela de Kramer.

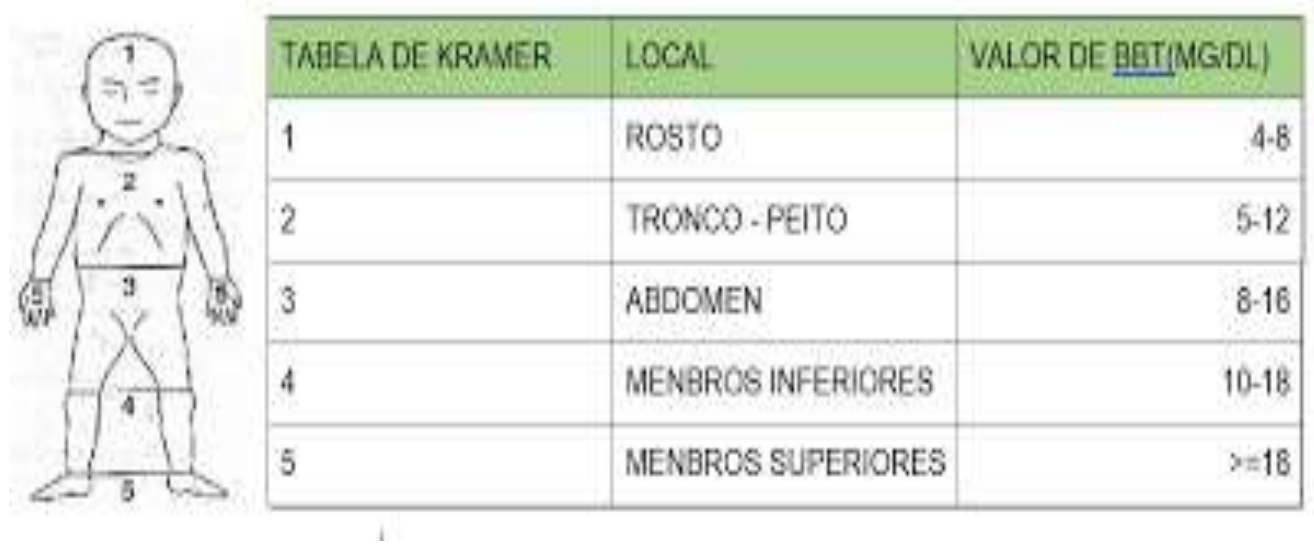

Fonte: Britto (2016). 
Baseado em nas possíveis complicações comumente encontradas nos neonatos ictéricos, há alguns diagnósticos que auxiliam o enfermeiro na Sistematização da Assistência de Enfermagem (SAE) destacando-se cinco diagnósticos de enfermagem de risco, sendo eles: o risco de volume de líquidos deficiente, o risco para integridade de pele prejudicada, o risco de integridade tissular prejudicada, o risco de desequilíbrio na temperatura corporal e o risco de motilidade gastrintestinal disfuncional (Alves, et. al., 2020).

A assistência de enfermagem ao neonato com hiperbilirrubinemia é de suma importância, pois acarreta em um menor tempo de internação, além de evitar possíveis sequelas irreversíveis. Torna-se necessário conscientizar-se quanto à identificação precoce dos fatores de risco para o recém-nascido, melhorando a condição de ajuda no tratamento. Diante exposto, se faz necessário que os profissionais da saúde, entre eles, os enfermeiros sejam qualificados e capazes de realizar o diagnóstico clínico de icterícia bem como proporcionar adequada assistência de enfermagem durante o tratamento (Gomes, Teixeira, Barrichello, 2010; Alves, et. al., 2020).

Contudo, apesar da relevância da patologia, ainda percebe-se a dificuldade dos enfermeiros na identificação precoce dos recém-nascidos com esta condição clínica e falha nas condutas de enfermagem que contribuam para eliminação ou conjugação bilirrubínica. Acredita-se que um dos fatores que contribuem para esta realidade seja a incompreensão na elaboração do diagnóstico de enfermagem. Diante do exposto, estudar a patologia, faz-se necessário para o aprimoramento dos cuidados oferecidos às crianças com essa condição (Dantas, 2017).

\section{Tratamento}

As formas de tratamento da icterícia neonatal incluem a transfusão de sangue ou plasma, exsanguineo transfusão, aplicação de albumina, fototerapia e drogas farmacológicas com a finalidade de acelerar o metabolismo e a excreção da bilirrubina, sendo que, a escolha dependerá do nível sérico, da incompatibilidade sanguínea, peso do RN, co-morbidades associadas, o tipo de icterícia, entre outros (Gomes, Teixeira \& Barichello, 2010; Sena, Reis, Cavalcante, 2015).

$\mathrm{O}$ tratamento adequado é definido após a determinação do tipo e intensidade da icterícia. A terapêutica utilizada pode ser fototerapia, exsanguineotransfusão e a administração de drogas adjuvantes, tais como metaloporfirinas inibidoras da hemeoxigenase, fenobarbital e imunoglobulina endovenosa sendo a fototerapia a intervenção mais amplamente usada no tratamento e prevenção da icterícia (Sacramento, et. al., 2017).

O tratamento de EXT foi considerado a primeira terapia com sucesso utilizada no tratamento para icterícia em estado grave em RNs, prevenindo, assim, seus efeitos tóxicos e a evolução para casos mais graves, como a encefalopatia ou kernicterus [6,12], por exemplo. Existem várias maneiras para realizar a EXT, sendo a mais comum em RN, a introdução de um cateter na veia umbilical o qual é empurrado até o interior da veia cava inferior, retirando parte do sangue do RN. Em seguida, assim que o sangue é removido em cada um dos ciclos, outro ciclo de sangue fresco de um doador escolhido é infundido no organismo do paciente. Dessa forma, a concentração de bilirrubina no sangue pode reduzir em até de 50\% do valor inicial (Lacerda, 2019).

Utiliza-se a fototerapia como tratamento padrão, sendo que o RN é exposto a uma luz de alta intensidade, a qual transforma a BI em uma molécula mais hidrossolúvel passível de ser excretada pelo rim ou fígado como prevenção da encefalopatia bilirrubínica. Sendo sua eficácia relacionada à intensidade, comprimento de onda da luz e da proporção de área de superfície corporal exposta à luz (Pinto, 2014; Romano, 2017).

Para definição do tipo de tratamento adequado deve-se observar diversos fatores, a fim de garantir que seja instituída a terapêutica mais eficaz. Preconiza-se que, primeiramente, observe-se o histórico materno e o histórico neonatal, analisar o aparecimento e a evolução da icterícia por meio do exame físico minucioso no RN e em seguida realizar a coleta de sangue do 
RN para análises. Deve-se, por fim, determinar se a hiperbilirrubinemia enquadra-se no tipo fisiológico ou patológico (Sacramento, et. al., 2017)

Segundo o Manual de Atenção à Saúde do Recém-nascido, os cuidados necessários ao RN em fototerapia são:

\begin{abstract}
"Verificar a temperatura corporal a cada três horas para detectar hipotermia ou hipertermia, e o peso diariamente; Aumentar a oferta hídrica, pois a fototerapia com lâmpada fluorescente ou halógena pode provocar elevação da temperatura, com consequente aumento do consumo de oxigênio, da frequência respiratória e do fluxo sanguíneo na pele, culminando em maior perda insensível de água; Proteger os olhos com cobertura radiopaca por meio de camadas de veludo negro ou papel carbono negro envolto em gaze; Não utilizar ou suspender a fototerapia se os níveis de BD estiverem elevados ou se houver colestase, para evitar o aparecimento da síndrome do bebê bronzeado, que se caracteriza pelo depósito de derivados de cobreporfirina no plasma, urina e pele; Cobrir a solução parenteral e o equipo com papel alumínio ou usar extensores impermeáveis à luz, pois a exposição de soluções de aminoácidos ou multivitamínicas ao comprimento de luz azul reduz a quantidade de triptofano, metionina e histidina; Adicionalmente, a solução de lipídeos é altamente susceptível à oxidação quando exposta à luz, originando hidroperóxidos de triglicérides citotóxicos; A prática da descontinuidade da fototerapia durante a alimentação, inclusive com a retirada da cobertura dos olhos, desde que a bilirrubinemia não esteja muito elevada" (Brasil, 2011b, p. 70).
\end{abstract}

O tratamento de fototerapia pode apresentar algumas alterações ao RN, tais como: diarreia, desidratação que ocorre em virtude da superfície corporal exposta em soma a demora na regulação do aleitamento materno, pré-disposição à hipertermia e à hipotemia em razão da exposição direta à luz bem como a falta de aquecimento em berços comuns, queimaduras, hemólise leve, síndrome do bebê bronzeado, plaquetopenia, danos retianos, erupções cutâneas e eritema (Gomes, Teixeira \& Barichello, 2010).

Dessa forma, para a realização adequada do modelo terapêutico, fototerapia, é de suma importância que a assistência à saúde prestada pela equipe de Enfermagem leve em consideração algumas questões, como por exemplo, a superfície corporal do RN exposta à luz, a distância apropriada entre o RN e a fonte luminosa, bem como os cuidados excepcionais com o paciente, desde a orientação sobre o tratamento para os pais e os cuidados deles perante o RN e a proteção ocular do recémnascido, juntamente com o controle dos sinais vitais e a avaliação do balanço hídrico, como forma de prevenção e para detectar precocemente possíveis alterações, com a finalidade de proporcionar uma terapia segura e eficaz ao RN (Machado, Samico, Brega, 2012).

Faz-se necessário o estabelecimento de um diálogo entre a equipe de enfermagem com os pais, visando informá-los a respeito do tratamento, do quadro clínico do RN e os procedimentos realizados na assistência à saúde do seu filho, uma vez que, a situação compromete a interação dos pais à criança e como forma de amenizar o sofrimento da família perante o RN com os olhos vendados e a exposição à luz (Araújo et. al., 2014).

De acordo com Sena, Reis e Cavalcante (2015) uma vez que o trabalho do enfermeiro em neonatologia em um caso de icterícia neonatal demanda vigilância, pois o paciente assistido é extremamente vulnerável e dependente da equipe de enfermagem, sendo assim, o enfermeiro possui como atribuição gerenciar a equipe de enfermagem, bem como as assistências à saúde, orientando que a equipe preste todo o cuidado necessário ao RN, com o enfoque da realização de um cuidado integral e humanizado.

Dessa forma, nota-se que o enfermeiro possui papel fundamental para a eficácia da fototerapia como propedêutica à hiperbilirrubinemia neonatal, visto que o mesmo, em conjunto à sua equipe, possui o maior contato com o paciente durante ao tratamento e sendo assim, dispõe a assistência à saúde de forma direta e consecutiva. Para isso, é de suma importância que os mesmos, tenham qualificação adequada para realizar o diagnóstico clínico, bem como, assegurar uma assistência de enfermagem efetiva (Sacramento et. al., 2017).

Segundo Gomes, Teixeira e Barrichello (2010) a situação solicita-se a atuação de uma equipe multidisciplinar, sendo que, demanda principalmente da equipe de enfermagem, a qual acompanha o paciente em tempo integral. Sendo assim, 
esperam-se profissionais com preparo qualificado para diagnosticar e intervir com rapidez e eficiência nas possíveis intercorrências, fazendo-se necessário a prestação de um cuidado à saúde, com embasamento técnico-científico, sistematizado e humanizado.

O sucesso da fototerapia depende da transformação fotoquímica da bilirrubina nas áreas expostas à luz. Essas reações alteram a estrutura da molécula de bilirrubina e permitem que os fotoprodutos sejam eliminados pelos rins ou pelo fígado, sem sofrerem modificações metabólicas. Portanto, o mecanismo de ação básico da fototerapia é a utilização de energia luminosa na transformação da bilirrubina em produtos mais hidrossolúveis. A bilirrubina absorve luz na região de 400 a $500 \mathrm{~nm}$. A luz emitida nesta faixa penetra na epiderme e atinge o tecido subcutâneo. Dessa forma, somente a bilirrubina que está próxima à superfície da pele (até $2 \mathrm{~mm}$ ) será afetada diretamente pela luz (Carvalho, 2001).

Recomenda-se a fototerapia para a redução de níveis séricos de bilirrubina em recém-nascidos. O que requer vários cuidados, pois nessa fase da vida a toxicidade a níveis altos pode acarretar em sequelas neurológicas irreversíveis. Para isso deve-se avaliar a eficácia que o tratamento fototerápico irá possuir (Finocchio, Tempesta, Ferreira, 2017).

As fontes de fototerapia comumente encontradas em hospitais têm por característica geral expor os RNs ao banho de luz em condições de isolamento, ou seja, sem o contato direto com as mães, e ainda possibilitando que a iluminação seja facilmente obstruída com o uso de cobertores e mantas juntos ao RN. Além disso, tendo-se fontes fixas cujos RNs são alvos dos feixes de luz, qualquer remoção ou obstrução involuntária do RN à iluminação torna-se um fator de risco, ou seja, de interrupção do tratamento fototerápico. Em adição, o calor gerado pela exposição à luz também pode levar os RNs a riscos de queimaduras e/ou aquecimento desnecessário (Lacerda, 2019).

Todos os recém-nascidos em fototerapia têm uma perda de líquido maior. Portanto, o balanço hídrico rigoroso e o exame físico completo são instrumentos para um cuidado com qualidade e com resultados futuros positivos. É de competência do enfermeiro a boa execução desses dois procedimentos. Cabe ao enfermeiro gerenciar o cuidado prestado ao recém-nascido ictérico, avaliando o estado clínico e a qualidade do tratamento indicado, principalmente quando se trata da fototerapia, em que há uma série de fatores de fundamental importância a serem examinados, tais como radiância do aparelho, tipo de fototerapia, condições do aparelho, temperatura, higienização, proteção ocular para o recém-nascido, distância do aparelho e recémnascido, entre outros. Isso se faz necessário para que de fato possa ser prestado um serviço de qualidade, eficiente e seguro ao cliente (Lopes, et. al., 2010).

\section{Conclusão}

A hiperbilirrubinemia conhecida como icterícia, constitui-se como uma síndrome frequente durante o período neonatal, tendo como característica processos fisiológicos e/ou patológicos, com apresentação da elevação de bilirrubina no sangue e consequentemente manifesta-se a coloração amarelada da pele, mucosa e líquidos orgânicos. Dessa forma, a atuação do enfermeiro se tem início na detecção precoce da icterícia, através do exame físico do RN e se estende durante a terapia proposta. Assim, a equipe de enfermagem é participativa desde o recebimento e preparação do recém-nascido para a terapêutica. Sendo que deve ser levado em consideração nesse diagnóstico e terapêutica a importância da humanização durante todo o processo por meio do profissional de enfermagem que assiste.

Conclui-se então que o tratamento adequado é definido após a determinação do tipo e intensidade da icterícia. A terapêutica utilizada pode ser fototerapia, exsanguineotransfusão e a administração de drogas adjuvantes, tais como metaloporfirinas inibidoras da heme-oxigenase, fenobarbital e imunoglobulina endovenosa sendo a fototerapia a intervenção mais amplamente usada no tratamento e prevenção da icterícia. 


\section{Referências}

Alves, A. L. N. et al. (2020). Diagnósticos de enfermagem em pacientes com icterícia neonatal. Brazilian Journal Of Development, 6(8), 57742-48. http://dx.doi.org/10.34117/bjdv6n8-254

Araújo, I. R. B. et al (2014). Nursing care of the newborns with neonatal jaundice: an integrative review. Revista de Enfermagem UFPI, (3)1, 120-4, https://doi.org/10.26694/reufpi.v3i1.1381

Brasil. (2011). Ministério da Saúde. Icterícia. In: Brasil. Ministério da Saúde. Atenção à saúde do recém-nascido: guia para os profissionais de saúde, volume 1. Brasília: Ministério da Saúde, p. 59-77.

Britto, W. M. (2016). Icterícia neonatal e enfermagem: conhecimentos e atitudes. 35 f. TCC (Doutorado) - Curso de Enfermagem, Faculdade de Educação e Meio Ambiente - Faema, Ariquemes. https://repositorio.faema.edu.br/bitstream/123456789/871/3/BRITO,\%20W.\%20M.\%20-\%20ICTER\%C3\%8DCIA $\%$ 20NEONATAL\%20E\%20ENFERMAGEM\%20CONHECIMENTOS\%20E\%20ATITUDES.pdf

Carvalho, M. (2001). Tratamento da icterícia neonatal. Jornal de Pediatria, 77(1), 71-81. http://www.jped.com.br/conteudo/01-77-s71/port.pdf

Dantas, A. V. V. C. (2017). Capacidade preditiva e prognóstica das características definidoras do diagnóstico de enfermagem icterícia neonatal. 71 f. Dissertação (Mestrado em Enfermagem) - Faculdade de Farmácia, Odontologia e Enfermagem, Universidade Federal do Ceará, http://www.repositorio.ufc.br/handle/riufc/23361

Finocchio, M. A. F., Tempesta, J. S. \& Ferreira, J. G. (2017). Proposta de um protótipo de iluminação fototerápica para o tratamento de icterícia. Revista Técnico-Científica do Crea, Paraná, 6(1), 1-19. https://www.brazilianjournals.com/index.php/BJHR/article/viewFile/17515/14217

Gomes, N. S., Teixeira, J. B. A., \& Barichello, E. (2010). Cuidados ao recém nascido em fototerapia: o conhecimento da equipe de enfermagem. Revista Eletrônica de Enfermagem, 12(2), 342-7, https://doi.org/10.5216/ree.v12i2.6507

Lacerda, G. S. (2019). Sistema fototerápico vestível para tratamento contínuo da icterícia neonatal. 100 f. Dissertação (Mestrado em Engenharia de Materiais) - Escola de Minas, Universidade Federal de Ouro Preto, Ouro Preto. http://www.repositorio.ufop.br/jspui/handle/123456789/11043

Lopes, V. M. et al. (2010). Icterícia neonatal e fototerapia: contribuição do enfermeiro para a eficácia do tratamento. Revista de Pesquisa Cuidado é Fundamental Online, 2(4), 1286-1296. https://www.redalyc.org/articulo.oa?id=505750833021

Machado, S. P. C., Samico, I. C., \& Braga, T. D. A. Conhecimento, atitude e prática sobre fototerapia entre profissionais de enfermagem de hospitais de ensino. Revista Brasileira de Enfermagem, 65(1), 34-41. https://doi.org/10.1590/S0034-71672012000100005

Moraes Filho I. M. et al. (2017). Checklist do recém-nascido: principais diagnósticos de enfermagem mediante intercorrências e susceptibilidade das mesmas no neonatal. Rev. Cient. Sena Aires., 6(1), 30-48. http://revistafacesa.senaaires.com.br/index.php/revisa/article/view/276

Nóbrega Júnior, G. S., Vieira, W. L., \& Guedes Júnior, J. A. (2019). Icterícia: uma doença comum entre os recém-nascidos. Brazilian Journal Of Health Review, Curitiba, 2(4), 2343-2350. https://www.brazilianjournals.com/index.php/BJHR/article/view/1696/1621

Pinto, S. L. (2014). Hiperbilirrubinemia neonatal: desenvolvimento de material e intervenção educacional para equipe de enfermagem. 21 f. Monografia (Especialização) - Curso de Curso de Especialização em Linhas de Cuidado em Enfermagem, Curso de Especialização em Linhas de Cuidado em Enfermagem, Santa Catarina. https://repositorio.ufsc.br/xmlui/handle/123456789/173213

Romano, D. R. (2017). Icterícia neonatal no recém-nascido de termo. 23 f. Dissertação (Mestrado) - Curso de Mestrado Integrado em Medicina, Instituto de Ciências Biomédica de Abel Salazar, Porto. https://repositorio-aberto.up.pt/bitstream/10216/109126/2/233122.pdf

Sacramento, L. C. A. et al. (2017). Icterícia neonatal: o enfermeiro frente ao diagnóstico e à fototerapia como tratamento. International Nursing Congress. Sergipe. https://eventos.set.edu.br/cie/article/viewFile/5705/2167

Santos, C. A. A. (2014). Cuidados de enfermagem em fototerapia. 17 f. Monografia (Especialização) - Curso de Programa de Pós-graduação em Enfermagem, Centro de Ciências da Saúde., Universidade Federal de Santa Catarina, Santa Catarina. https://repositorio.u fsc.br/xmlui/bitstream/handle/123456789/171954/Carlos\%20Alberto\%20Anselmo\%20dos\%20Santos\%20-\%20SMNL.pdf?sequence=1\&isAllowed=y

Sarli, Y. O. (2018). Associação entre o tempo de clampeamento do cordão umbilical e icterícia neonatal precoce em recém nascidos a termo. 72 f. Dissertação (Mestrado) - Curso de Mestrado em Ciências da Saúde, Universidade Santo Amaro, São Paulo. http://dspace.unisa.br/handle/123456789/183

Sena, D. T. C., Reis, R. P., \& Cavalcante, J. B. N. (2015). A importância da atuação do enfermeiro no tratamento da icterícia neonatal. Revista Eletrônica Estácio Saúde, Santa Catarina, 4(2), 160-170. http://hdl.handle.net/123456789/183

Sousa, A. B. M., \& Sena, D. S. L. (2016). Hiperbilirrubinemia neonatal: considerações sobre fisiopatogenia, modalidades terapêuticas e complicações. Revista FACID, Teresina, 8(2), 17-25. https://repositorio.faema.edu.br/bitstream/123456789/871/3/BRITO,\%20W.\%20M.\%20-\%20ICTER\%C3\%8DCIA \%20NEONATAL\%20E\%20ENFERMAGEM\%20CONHECIMENTOS\%20E\%20ATITUDES.pdf

Teixeira, A. A. (2014). O enfermeiro frente à icterícia neonatal. 32 f. TCC (Graduação) - Curso de Enfermagem, Faculdade de Educação e Meio Ambiente Faema, Ariquemes. https://repositorio.faema.edu.br/handle/123456789/934 